\title{
Textbook of OPERATIVE DENTISTRY
}
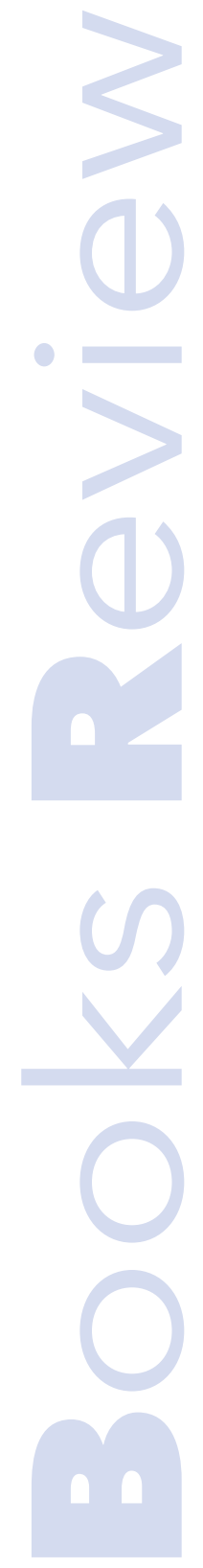

\section{Nisha Garg, Amit Garg}

Publisher: Jaypee Brothers Medical Publishers (P) Ltd

Language: English

ISBN: 978-93-5025-939-9

Edition: 2/e

Publish Year: 2013

Pages: 624

Price: $f 26.00$

The "Textbook of Operative Dentistry" by Nisha Garg and Amit Garg is divided into 32 chapters addressing the topic of clinical practice of operative dentistry from the classical to the modern trends.

After specifying the indications and purpose of operative dentistry, the authors describe the pathology of dental caries, illustrating the cutting instruments from hand, ultrasonic ones to laser instruments, principles of tooth preparation, matricing and tooth separation.

The textbook presents the patient evaluation, diagnosis, treatment planning, and components of the oral environment, the equipment needed for isolation and the methods used for gingival tissue management.

Further on it provides considerations on patient treating and the sitting arrangement of the operator and assistant, as well as infection control, cross infection, disinfection to infection control, and also the checklist during pretreatment, chairside and post treatment period.

The subsequent chapters continue in the same style, enlarging upon methods of pain control in dental clinic, materials and methods used for pulp protection interim restorations for intracoronal and extracoronal preparations.

The book covers adhesive dentistry, indications for use, advantages and adhesion mechanism; indications and contraindications of composites, tooth preparation, direct and indirect composite restorations, completed in the next chapter entitled "Esthetics and Operative Dentistry".

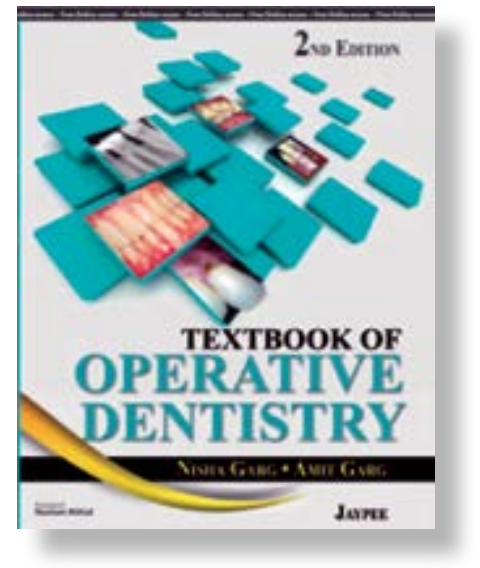

Following the plastic restorations, the rigid restorations (amalgam, pin amalgam restorations, direct gold fillings and cast metal restorations), indications and contraindications as well as the principles and steps of tooth preparation are presented.

The book covers the incidence and the latest treatment strategies for tooth hypersensitivity, discolored teeth and cervical lesions.

Recent trends of minimal intervention dentistry and conscious efforts to practice maximum conservation as per today's need of the patients, nanotechnology advantages and its applications in dentistry are convincingly described in the last chapters.

After going through this book, the reader will be able to identify indications and contraindications of different dental materials, apply modern pulp protective regimens and select suitable restorative materials for restoration of teeth.

All the chapters of the textbook are drafted in a simple manner, and incorporate more than 1200 colored illustrations along with graphs, flow charts and tables. It is a reference book on operative dentistry with a classical approach, but with new trends for every dental professional and student. 